\title{
Inovação em saúde e internet das coisas (IoT): Um panorama do desenvolvimento científico e tecnológico
}

\author{
Claudia Marisa Rosa ${ }^{I}$ \\ http://orcid.org/0000-0002-4144-0577
}

Paulo Augusto Ramalho de Souza ${ }^{I I}$

http://orcid.org/0000-0002-4046-2811

Joaquim Manoel da Silva ${ }^{I I I}$

http://orcid.org/0000-0002-4046-1405

${ }^{I}$ Universidade Federal de Mato Grosso, MT, Brasil.

Mestre em Propriedade Intelectual e Transferência de Tecnologia para a

Inovação.

${ }^{\text {II }}$ Universidade Federal de Mato Grosso, MT, Brasil.

Doutor em Administração pela Universidade Municipal de São Caetano do Sul (USCS).

III Universidade do Estado de Mato Grosso, MT, Brasil.

Doutor em Genética e Biologia Molecular - ênfase em Bioinformática pela

Universidade Estadual de Campinas (UNICAMP).

http://dx.doi.org/10.1590/1981-5344/3885

Esta pesquisa pautou-se na análise do desenvolvimento científico e tecnológico acerca da Internet das Coisas (IoT) aplicada ao ambiente da saúde na perspectiva da inovação, tendo como insumo artigos científicos e documentos de patentes relacionados ao tema.A prospecção realizada a partir dos critérios delimitados possibilitou o mapeamento, nos últimos dez anos, de 217 artigos e 177 patentes relacionados à temática, em nível global. Os dados foram analisados utilizando-se os 
parâmetros da análise bibliométrica seguida da análise de conteúdo,permitindo a identificação dos autores com maior produtividade (Lei de Lotka), a vinculação destes autores, a dispersão histórica das publicações e dos periódicos que difundem o assunto (Lei de Bradford), as abordagens predominantes das publicações (Lei de Zipf)e os países e as áreas de destaque em publicações científicas e tecnológicas. O panorama delineado aponta que o desenvolvimento da temática ocorreu a partir de 2010 liderado por países asiáticos, com uma produção científica mais desconcentrada e predominantemente vinculada às Universidades, enquanto a produção tecnológica apresenta maior concentração e vinculação com empresas, porém, com convergência no discurso comum. Dentre as tendências identificadas, sobressai o monitoramento remoto de pacientes.

Palavras-chave: Inovação; Saúde; Internet das coisas; Desenvolvimento científico; Desenvolvimento tecnológico.

\section{Innovations in health care and the internet of things (IOT): An overview of technological and scientific research}

This study provides an analysis of the scientific and technological research on the applications of the Internet of Things (IOT) within the field of health care. It focuses on innovations and is based on scientific articles and patent documentation. The search for data, which was global in scope, was based on specific criteria and enabled a mapping of 217 articles and 117 patents involving the IoT applied to health care over the last 10 years. Bibliometric analysis was applied to the data, followed by content analysis. The analyses applied herein identified the most productive authors (Lotka's law), their affiliations, the distribution of publications over time, the core journals in which this topic is distributed (Bradford's law), the predominant approaches used in the publications (Zipf's law), and the countries and regions in which this scientific and technological research is most 
commonly performed. This topic was found to have been most heavily researched since 2010, and this research is led by countries in Asia. Scientific research on this topic is predominantly affiliated with universities, while technological research on this topic is more likely to be affiliated with corporations, though their discussions and conclusions are consistent. Among the topics of study identified, the most frequent was the remote monitoring of patients.

Keywords: Innovation; Health care; The Internet of things; Scientific research; Technological development.

Recebido em 07.03.2019 Aceito em 19.08.2020

\section{Introdução}

Desde o advento da rede de comunicação que deu origem a internet, denominada Advanced Research Projects Agency Network (ARPANET)(FIDLER; CURRIE, 2015) a constante evolução da Tecnologia da Informação e Comunicação (TIC) tem contribuído significativamente no surgimento de inovações que impactam a vida dos indivíduos e das organizações, resultantes de contínuos esforços científicos e tecnológicos.

É neste contexto evolutivo que surge a Internet das Coisas, ou Internet of Things (IoT), baseada em uma infraestrutura de interconexão e interação de objetos que tendem a assumir o controle de uma diversidade de ações rotineiras sem a necessidade de intervenção humana em seu comando (SANTAELLA et.al, 2013). Essa integração do mundo físico e virtual se dá por meio da coleta, processamento e análise de dados gerados por rede de sensores, atuadores, processadores e computadores conectados à internet (ALBERTIN; ALBETIN, 2017) e (LAPLANTE; LAPLANTE, 2016).

As possibilidades e perspectivas do uso da IoT são abrangentes e difusas, desde aplicações mais triviais até as invenções futuristas e desafiadoras do senso comum, o que requer a ampliação do campo de estudo visando a consolidação de questões como: quais as características do desenvolvimento científico e tecnológico associado a aplicabilidade da IoT no ambiente da saúde? Com o propósito de contribuir com este campo de estudo, o objetivo nesta pesquisa é analisar o estado da arte do cenário científico e tecnológico que suporta as bases do desenvolvimento 
da IoT aplicada à saúde e que contribui para a inovação neste ambiente de intensa Pesquisa e Desenvolvimento (P\&D).

Os avanços tecnológicos no ambiente da saúde apontam para a denominada "Saúde 4.0", uma alusão a IV Revolução Industrial, onde o universo da tecnologia e da saúde se unem utilizando-se da computação em nuvem e IoT para prover dentre outras aplicações a digitalização de dados, sobretudo de prontuários médicos, interconectividade entre máquinas e comandos,banco de dados mais eficientes, resultando em maior agilidade nas respostas médicas e maior autonomia do paciente em relação à sua saúde (ENGELMANN, 2018).

Para análise do desenvolvimento científico e tecnológico que envolve o paradigma da IoT aplicada à saúde em âmbito global realizou-se estudo bibliométrico da produção científica, por meio de artigos indexados em periódicos científicos disponíveis na base de dados Scopus ${ }^{\circledR}$ e da produção tecnológica mediante prospecção de patentes utilizando o software Questel Orbit ${ }^{\circledR}$.

De acordo com Spinak (1998) a bibliometria estuda a organização dos setores científicos e tecnológicos a partir de fontes bibliográficas e de patentes para identificar os atores, suas relações e suas tendências. Neste mesmo sentido, Araújo (2006) indica que a bibliometria consiste na técnica quantitativa e estatística de medição dos índices de produção e disseminação do conhecimento científico.

A partir da análise dos artigos e patentes foi possível mapear as características da produção cientifica e tecnológica, acerca da temática da IoT no ambiente da saúde, identificado os autores com maior produtividade e a vinculação destes, a evolução histórica das publicações, os periódicos e áreas que mais difundem o assunto, as abordagens predominantes das publicações, além dos países e que se destacam na publicação dos artigos e patentes.

\section{Revisão bibliográfica}

O referencial teórico utilizado na abordagem do paradigma da IoT aplicada à saúde baseou-se em um arcabouço conceitual e teórico com a finalidade dedar suporte a reflexão sobre o tema e a delimitação dos métodos de pesquisa e apresentação dos resultados.

Internet das coisas (IoT) pode ser conceitualmente definida como uma infraestrutura de rede global dinâmica baseada em protocolos de comunicação padrão e interoperáveis que atribui identidade e interface inteligente as "coisas" físicas e virtuais, integrando-as perfeitamente a redes de informações(VERMESAN; FRIESS, 2011).

Enquanto inovação é definida pelo Manual de Oslo (Finep, 2005) como a implementação, no âmbito de uma organização, de um produto novo ou significativamente melhorado, ou de um novo processo, método 
de marketing ou organizacional. É uma definição bastante ampla, que abrange como atividade de inovação todas as etapas que conduzem à implementação da inovação, sejam elas científicas, tecnológicas, organizacionais, financeiras e comerciais (FINEP, 2005).

A compreensão do papel da inovação nos diferentes ambientes é condição para o desenvolvimento, segundo Atun e Sheridan (2007) as sociedades que se afastaram da inovação e do desenvolvimento tecnológico falharam na capacidade de sustentar suas populações.

Neste contexto, Atun e Sheridan (2007) enfatiza que:

Understanding the nature of innovation in the life sciences is critical to creating innovation ecosystems that combine an appropriate balance of incentives, rewards and regulations that encourage the innovation process but also the uptake and difusion of innovations once these reach the market place.

Developing such an understanding requires a careful examination of the nature of innovation in the life sciences, the innovation process that spans academic institutions, healthcare systems and multiple industrials organizations[...]. (ATUN; SHERIDAN, 2007, p. VII).

Ao revisar a literatura é possível verificar a diversidade de uso da IoT no ambiente da saúde, com inovações para simplificação de processos, otimização de tempo e recursos, redução de erros por intervenção humana, personalização de tratamentos e disponibilização de informações para prevenção e controle de doenças, sempre na perspectiva da melhora da qualidade de vida e do bem-estar da sociedade.

Para Oliveira e Silva (2017) essas inovações se dividem em três principais categorias. A primeira delas inclui diagnóstico e monitoramento remoto de pacientes por meio de dispositivos vestíveis, os wearables, e sensores que podem monitorar desde a qualidade do ar, à ingestão de medicamentos e até mesmo a detecção de quedas e acionamento de unidades de emergência. A segunda está relacionada à telemedicina por meio de teleconsultas e acompanhamento médico a distância. Já a terceira categoria abrange as plataformas que auxiliam pacientes a mudarem hábitos e comportamentos na adoção de um estilo de vida mais saudável.

As tecnologias decorrentes da aplicação de IoT no ambiente da saúde experimentam altas taxas de crescimento em um cenário de inúmeras possibilidades. Estudo da Accenture (2017), baseados em dados do e Marketer, aponta que os investimentos estimados em IoT para cuidados de saúde alcançará a marca de 163 bilhões de dólares até o ano de 2020, registrando crescimento anual de aproximadamente $38,1 \%$ entre 2015 e 2020. Este estudo aponta ainda que nos próximos anos o 
ambiente da saúde é o número 1 entre os 10 principais setores para desenvolvimento de aplicativos baseado em IoT.

O cenário posto, com amplas perspectivas,assinala a necessidade de mapear e analisar a produção científica e tecnológica que fornece as bases do desenvolvimento da IoT aplicada à saúde. A prospecção tecnológica utilizada no mapeamento pode ser compreendida, segundo Mayerhoff (2008), como "um meio sistemático de mapear desenvolvimentos científicos e tecnológicos futuros capazes de influenciar de forma significativa uma indústria, a economia ou a sociedade como um todo". Quanto a análise, conforme Freitas (2018), a produção científica pode ser avaliada em várias instâncias e de vários modos, desde a análise de produtos resultantes de atividade científica como patentes, inventos, relatórios, artigos, dissertações e teses, até as avaliações formais e institucionalizadas desenvolvidas por entidades científicas e/ou governamentais.

Os periódicos científicos, utilizados como fonte de dados neste estudo, são destacados por Gil (2002) como o mais importante mecanismo para a comunicação científica, "graças a eles é que vem se tornando possível a comunicação formal dos resultados de pesquisas originais e a manutenção do padrão de qualidade na investigação científica".

\section{Procedimentos metodológicos}

Quanto aos métodos,trata-se de pesquisa aplicada descritiva, elaborada a partir da abordagem quantitativa de dados bibliográficos e documentais oriundos de fontes secundárias relacionadas a produção científica e tecnológica envolvendo IoT no ambiente da saúde. Para Gil (2008) as pesquisas descritivas têm como objetivo principal a descrição das características de uma determinada população ou fenômeno utilizando-se de técnicas padronizadas de coleta de dados.

Por meio de prospecção exploratória realizou-se o mapeamento da produção científica através de publicações de artigos em periódicos científicos indexados à base de dados $S \operatorname{copus}{ }^{\circledR}, \mathrm{e}$ para a produção tecnológica utilizou-se o software de localização e exploração de patentes, Questel Orbit ${ }^{\circledR}$. Os dados pertinentes ao estudo foram exportados para a ferramenta Excel ${ }^{\circledR}$ e para o software $N$ Vivo ${ }^{\circledR} 10$ for Windows visando a análise, realizada em duas etapas, uma bibliométrica e a outra exploratória de conteúdo a partir da frequência e agrupamento dos principais termos, presentes nos títulos e resumos dos artigos e patentes.

$\mathrm{Na}$ Tabela 1 são apresentados os critérios de buscas aplicados, a partir da delimitação dos descritores em inglês "Internet of things", "IoT" e "Health", em ambas as bases, visando filtrar os resultados ao propósito do estudo. 
Tabela 1 - Critérios utilizados nas buscas de artigos e de patentes

\begin{tabular}{ll}
\hline & \multicolumn{1}{c}{ Critérios de buscas } \\
\hline Tipo de publicação & Artigos; Patentes \\
\hline Booleano & OR; AND \\
\hline Truncagem & 1 termo (health) \\
\hline Abrangência & Título \\
\hline Período de cobertura & 2008 a 2018
\end{tabular}

Fonte: Elaborado pelos autores.

Para desenvolvimento da análise bibliométrica, tomou-se por base os pressupostos das leis da bibliometria, a Lei de Lotka (1926) referente a análise da produtividade dos autores e a contribuição destes para o desenvolvimento científico em suas respectivas áreas de conhecimento (lei do quadrado inverso); Lei de Bradford (1934)que tem o propósito de conhecer a dispersão do conhecimento publicado em periódicos científicos sobre determinado assunto (lei da dispersão) e a Lei de Zipf (1949) que trata da distribuição da frequência de palavras, possibilitando mensurar a abordagem de um texto pela frequência das palavras mais usadas (princípio do menor esforço em terminologias) (Rodrigues; Viera, 2016) e (Araújo, 2006).

\section{Resultados e discussões}

A prospecção da produção científica e tecnológica, realizada em novembro de 2018, a partir da combinação de descritores e critérios resultou em 217 artigos e177 registros de patentes observada a primeira publicação.

Como o escopo central deste estudo pautou-sena análise do panorama científico e tecnológico relacionado aIoT aplicada à saúde e as inovações resultantes deste mecanismo, decidiu-se por além dos pressupostos das leis de Lotka, Bradford e Zipf apresentar informações que contribuam no delineamento do estado da arte da temática, priorizando um cenário comparativo delimitado em um recorte temporal de dez anos,associado ao recorte dos dez principais resultados para cada item em análise, a começar pelos países que se destacam na publicação dos artigos e na proteção das patentes, apresentados na Tabela 2. 
Tabela 2 - Comparativo dos países de publicação dos artigos e proteção das patentes

\begin{tabular}{l|c|l|c}
\hline \multicolumn{1}{c|}{ Publicação do artigo } & no Artigos & \multicolumn{1}{|c}{ Proteção da patente } & no Patentes \\
\hline Índia & 54 & China & 103 \\
China & 40 & Coreia do Sul & 15 \\
Coreia do Sul & 30 & Índia & 10 \\
\hline Estados Unidos & 29 & Estados Unidos & 5 \\
Reino Unido & 14 & Reino Unido & 3 \\
\hline Arábia Saudita & 10 & Taiwan & 3 \\
Taiwan & 10 & Japão & 2 \\
Austrália & 9 & Escritório Europeu de Patentes (EP) & 2 \\
Paquistão & 9 & Tratado de Cooperaçãode Patentes (PCT) & 2 \\
Suécia & 8 & & \\
\hline Outros (35 Países) & 88 & & \\
\hline
\end{tabular}

Fonte: Elaborado com base em Scopus e Questel Orbit.

Nota-se a convergência entre os países com maior publicação de artigos e proteção de patentes, destacando-se a Índia e a China, respectivamente.A dispersão das publicações de artigos é mais perceptível, porém no âmbito das patentes o Tratado de Cooperação de Patentes (PCT) abrange 152 países, de acordo com a World Intellectual Property Organization (WIPO), tal qual o Escritório Europeu de Patentes (EP) com cobertura na Europa, cabendo ao requerente eleger em quais destes países requer a proteção.

Percebe-se na análise estatística a existência de colaboração entre autores de diferentes países na elaboração de artigos, enquanto a proteção das patentes está vinculada a situação legal do registro, conforme demonstrado no Gráfico 1. 


\section{Gráfico 1 - Situação legal das patentes}

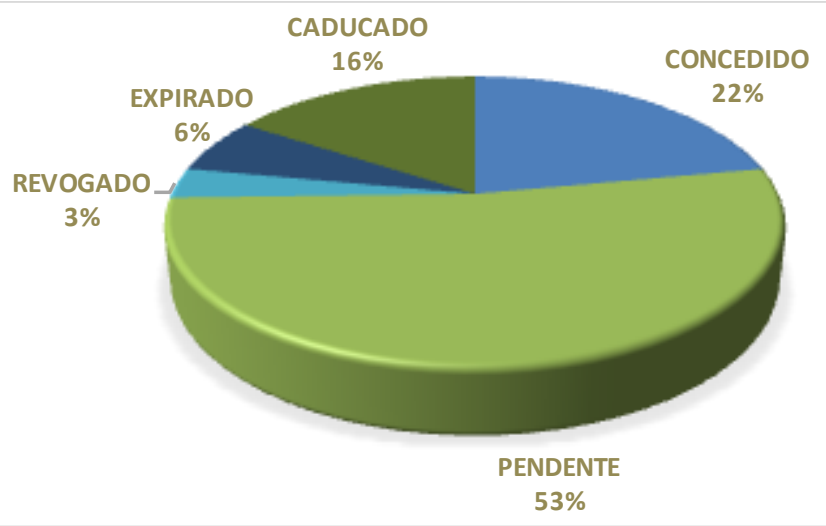

Fonte: Elaborado com base em Questel Orbit.

Numericamente, das 177 patentes mapeadas, 132 estão ativas, presentes no Gráfico 1 com status de "concedido", assinalando a concessão da proteção, e "pendente", indicando a fase de análise, enquanto 45 estão extintas.

A partir da análise identificou-se os principais grupos de classificação das patentes com base na Classificação Internacional de Patentes (IPC) da World Intellectual Property Organization (WIPO), apresentados na Tabela 3 , considerando a existência de registros pertinentes a mais de um grupo de classificação.

Tabela 3 - Grupos de patentes com base na Classificação Internacional de Patentes (IPC)

\section{Principais Grupos IPC}

$\%$ Patentes

(A61B-005) Medição para fins de diagnóstico; Identificação de pessoas

$27,2 \%$

(G06F-019) Computação digital ou equipamento ou métodos de processamento de dados especialmente adaptados para aplicações específicas

(G06Q-050) Sistemas ou métodos especialmente adaptados para setores de atividade específicos, e.g. serviços públicos ou turismo

(H04L-029) Arranjos, aparelhos, circuitos ou sistemas [...]

(A61B-090) Instrumentos, implementos ou acessórios especialmente adaptados para cirurgia ou diagnóstico $[\ldots]$

(G05B-019) Sistemas de controle de programa

(G16H-050) TIC especialmente adaptado para diagnóstico médico, simulação médica ou mineração de dados médicos; TC especialmente adaptado para detectar, monitorar ou modelar epidemias ou pandemias 
(A47G-019) Bancada de serviço

(A61N-005) Terapia de radiação

(B61K-009) Medidores de perfil de veículos ferroviários; detectar ou indicar superaquecimento de componentes; Aparelhos de locomotivas ou carros para indicar seções de pista defeituosas; Projeto geral de veículos de registro de pista

\section{Fonte: Elaborado com base em Questel Orbit.}

Observa-se uma concentração de aplicações de IOT no âmbito de diagnósticos, identificação de pessoas, sistemas e métodos de processamento de dados, seguido por soluções em aparelhos, instrumentos ou acessórios adaptados para procedimento cirúrgicos e diagnósticos.

Além dos grupos de classificação, identificou-se as principais famílias de patentes, por ordem de importância destacam-se Tecnologia Médica; Comunicação Digital; Métodos de TI para Gerenciamento; Tecnologia de Computadores; Controle; Mobiliários e Jogos; Medição; Telecomunicações; Maquinários Elétricos, Aparelhos e Energia; e Engenharia Civil.

Quanto a produção científica, Ciência da Computação e Engenharia se sobressaem entre as áreas de conhecimento com maior publicação de artigos na temática da IoT no ambiente de saúde, conforme o Gráfico 2, considerando a categorização das áreas de conhecimento utilizada pela base de dados Scopus $®$.

\section{Gráfico 2 - Publicações de artigos por área de conhecimento}

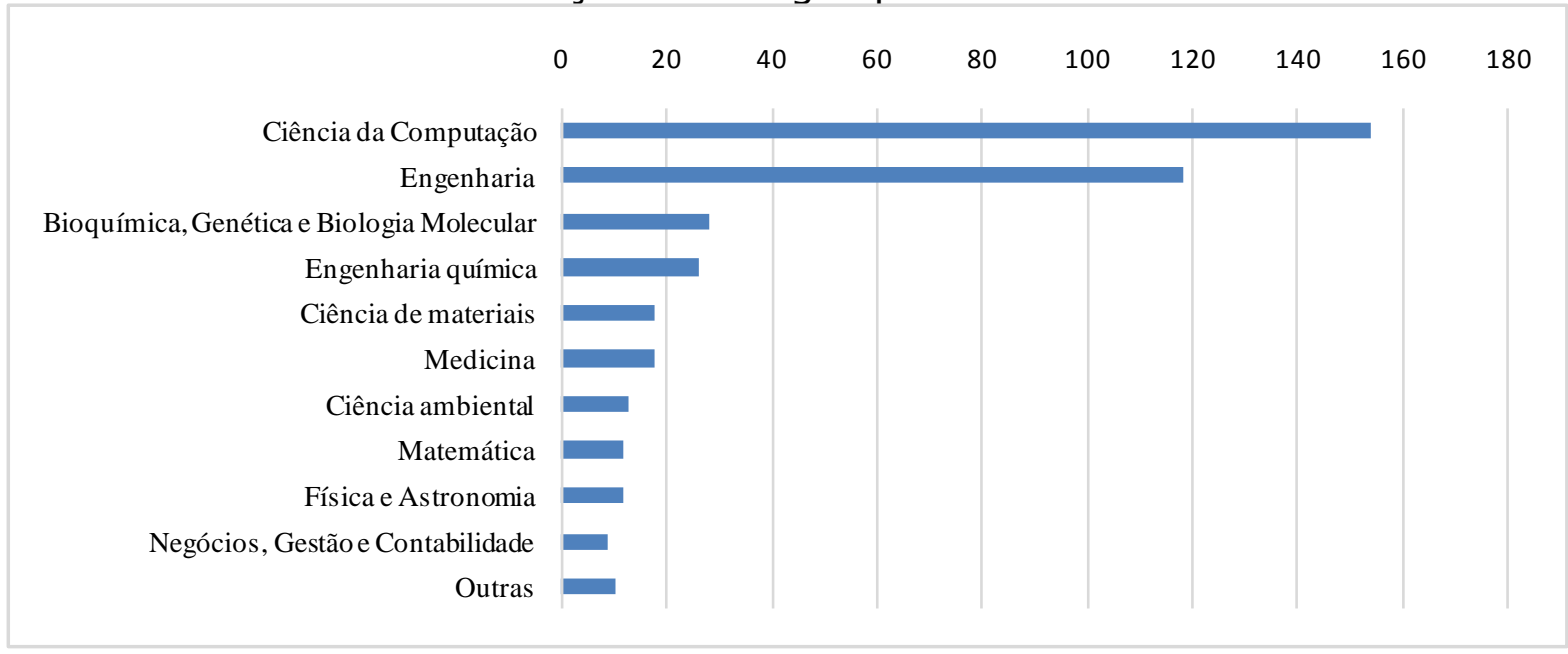

Fonte: Elaborado com base em Scopus. 
$\mathrm{Na}$ categoria "Outras", em menor número, além de publicações multidisciplinares estão presentes as áreas de Ciências Sociais; Farmacologia, Toxicologia e Farmacêutica; Profissões de Saúde; Ciências da Decisão; Química; Psicologia; Ciências Agrárias e Biológicas; Energia; Imunologia e Microbiologia; Artes e Humanidades; e Ciências da Terra e Planetárias.

Seguindo a análise da produção científica, sob a perspectiva da Lei de Lotka para os autores, e da Lei de Bradford para os periódicos elaborou-se a Tabela 4. Para manutenção do padrão bibliométrico desconsiderou-se a coautoria, acompanhando os parâmetros utilizados por Oliveira e Gracio (2011), Santos, Souza e Pereira (2014) e Rodrigues e Viera (2016).Os resultados contemplam as variações na grafia do nome de um mesmo autor, conforme consta no periódico.

Tabela 4 - Comparativo dos periódicos e dos autores com maior produtividade

\begin{tabular}{l|c|c|c}
\hline \multicolumn{1}{c}{ Periódicos } & no Artigos & \multicolumn{1}{c}{ Autores } & no Artigos \\
\hline $\begin{array}{l}\text { International Journal of Engineering and } \\
\text { Technology (UAE) }\end{array}$ & 12 & Lee, B.M. & 4 \\
\hline Future Generation Computer Systems & 11 & Bhuiyan, M.Z.A. & 3 \\
\hline IEEE Access & 11 & Kumar, P.M. & 3 \\
\hline $\begin{array}{l}\text { IEEE Internet of Things Journal } \\
\text { IEEE Communications Magazine }\end{array}$ & 6 & Laplante, P.A. & 3 \\
\hline $\begin{array}{l}\text { International Journal of Bio Science and } \\
\text { Bio Technology }\end{array}$ & 5 & Sood, S.K. & 3 \\
\hline International Journal of Smart Home & 5 & Yang, Y. & 3 \\
\hline Journal of Medical Systems & 5 & Yeh, K.H. & 3 \\
\hline AppliedSciencesSwitzerland & 4 & Abawajy, J.H. & 3 \\
\hline $\begin{array}{l}\text { International Journal of Applied } \\
\text { Engineering Research }\end{array}$ & 4 & Abhishek, A. & 2 \\
\hline Outros (104 Periódicos) & & & 2 \\
\hline
\end{tabular}

Fonte: Elaborado com base em Scopus.

Nota-se uma grande dispersão no número de periódicos e autores, indicando a desconcentração das publicações científicas que abordam o assunto. Para compreensão da vinculação dos autores, elaborou-se a Tabela 5, associando um comparativo com as instituições requerente das patentes. 
Tabela 5 - Vinculação dos autores de artigos e requerentes de patentes por instituição

\begin{tabular}{l|c|l|c}
\hline \multicolumn{1}{|c|}{ Autores de artigos } & no Artigos & \multicolumn{1}{c}{ Requerentes de patentes } & $\begin{array}{c}\text { no } \\
\text { Patentes }\end{array}$ \\
\hline $\begin{array}{l}\text { VelloreInstituteof Technology } \\
\text { King SaudUniversity }\end{array}$ & 18 & Aserie Marcus Linval & 5 \\
\hline $\begin{array}{l}\text { Pennsylvania StateUniversity } \\
\text { The KTH Royal Institute of }\end{array}$ & 4 & NanjingWeilvBiotechnology & 5 \\
\hline $\begin{array}{l}\text { Technology } \\
\text { BlekingeTekniskaHogskola }\end{array}$ & 4 & ShenzhenAlmsound Technology & 3 \\
\hline ChineseAcademyofSciences & 4 & $\begin{array}{l}\text { ShenzhenCannice Technology } \\
\text { University Coop. Foundation }\end{array}$ & 3 \\
\hline UniversityofGachon & 4 & AangelKids & 3 \\
\hline Universityof Nagoya & 3 & Anychecklnformation Technology & 2 \\
\hline MinistryofEducation China & 3 & Aserie Marcus & 2 \\
\hline SASTRA University & 3 & Haiersoft & 2 \\
\hline Outros (150 Instituições) & 232 & Outros (20 Instituições) & 35 \\
\hline
\end{tabular}

Fonte: Elaborado com base em Scopus e Questel Orbit.

Observa-se que não há convergência entre as instituições relacionadas a produção científica e as detentoras da produção tecnológica, a produção científica é predominantemente vinculada às universidades, enquanto a produção tecnológica às empresas. A dispersão de instituições na produção científica é mais evidente diante da vinculação dos coautores, enquanto a produção tecnológica tem maior característica de independência de vínculo institucional, decorrente dos autores/inventores autônomos.

Alinhado aos parâmetros da Lei de Bradford, elaborou-se a evolução histórica da produção científica e tecnológica ao longo dos anos, apresentada no gráfico 3, considerando para as patentes a primeira publicação. 
Gráfico 3 - Dispersão do número de artigos e patentes por ano de publicação

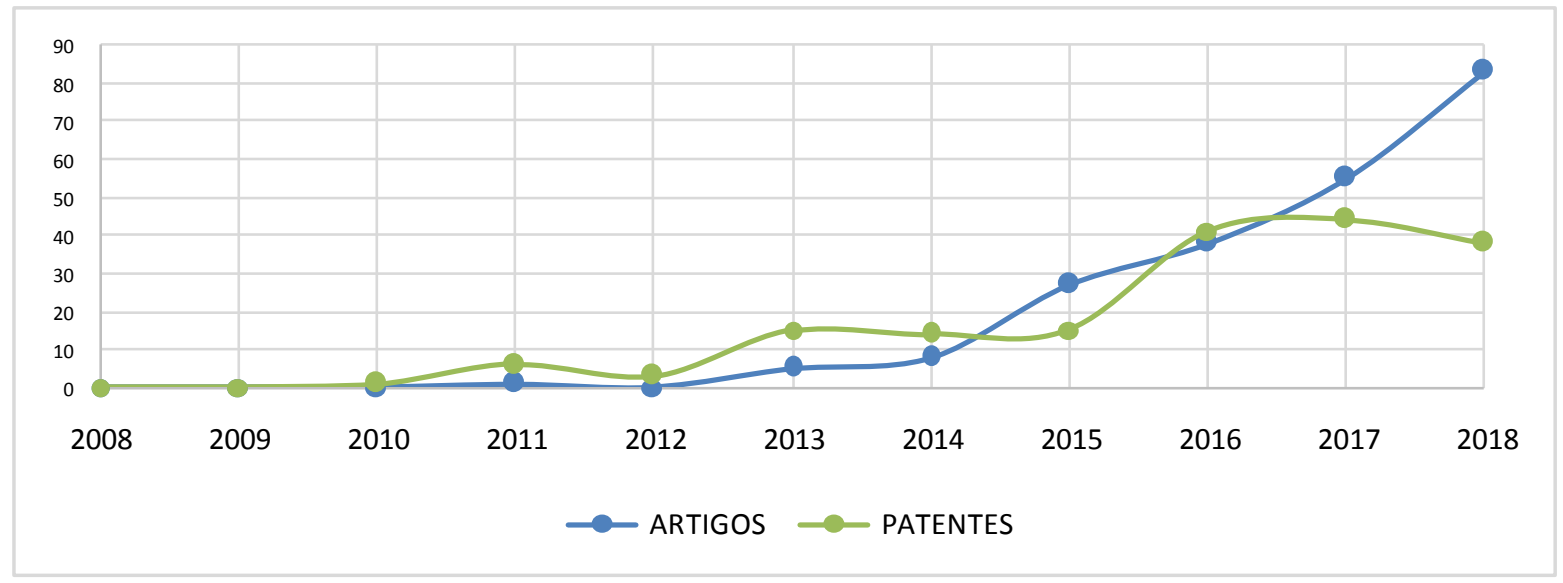

Fonte: Elaborado com base em Scopuse Questel Orbit.

Observa-se que primeira publicação se deu no âmbito das patentes, em 2010, e uma dispersão convergente até o ano de 2016, seguido por leve declínio na publicação de patentes e um crescimento acentuado na publicação de artigos. O comportamento apresentado reforça a dinâmica de proteger para depois publicar atendendo aos requisitos de novidade absoluta, a despeito das exceções aplicáveis. Isso posto, somado ao fato de que em regra a primeira publicação do pedido de patente ocorre 18 meses após efetivado o depósito, tem-se a compreensão da variação negativa na produção tecnológica a partir de 2016.

Para compreensão da abordagem predominante da IoT no ambiente da saúde, a partir dos pressupostos da Lei de Zipf, utilizando-se do software NVivo ${ }^{\circledR}$ 10for Windows, realizou-se a análise dos títulos e resumos dos artigos e patentes, por entender que estas estruturas compilam a expressão do texto completo. Com base na técnica de contagem de frequência de palavras, optou-se por delimitar em 30 resultados,associado a cinco clusters de agrupamento dos discursos comuns, com isso foi possível identificar os principais termos relacionados a temática em nível global, presentes na Figura 1 referente aos artigos $(C A)$, e na Figura 2 às patentes $(C P)$, apresentadas em sequência para fins comparativos. 
Figura 1 - Agrupamento dos discursos comuns aos artigos.

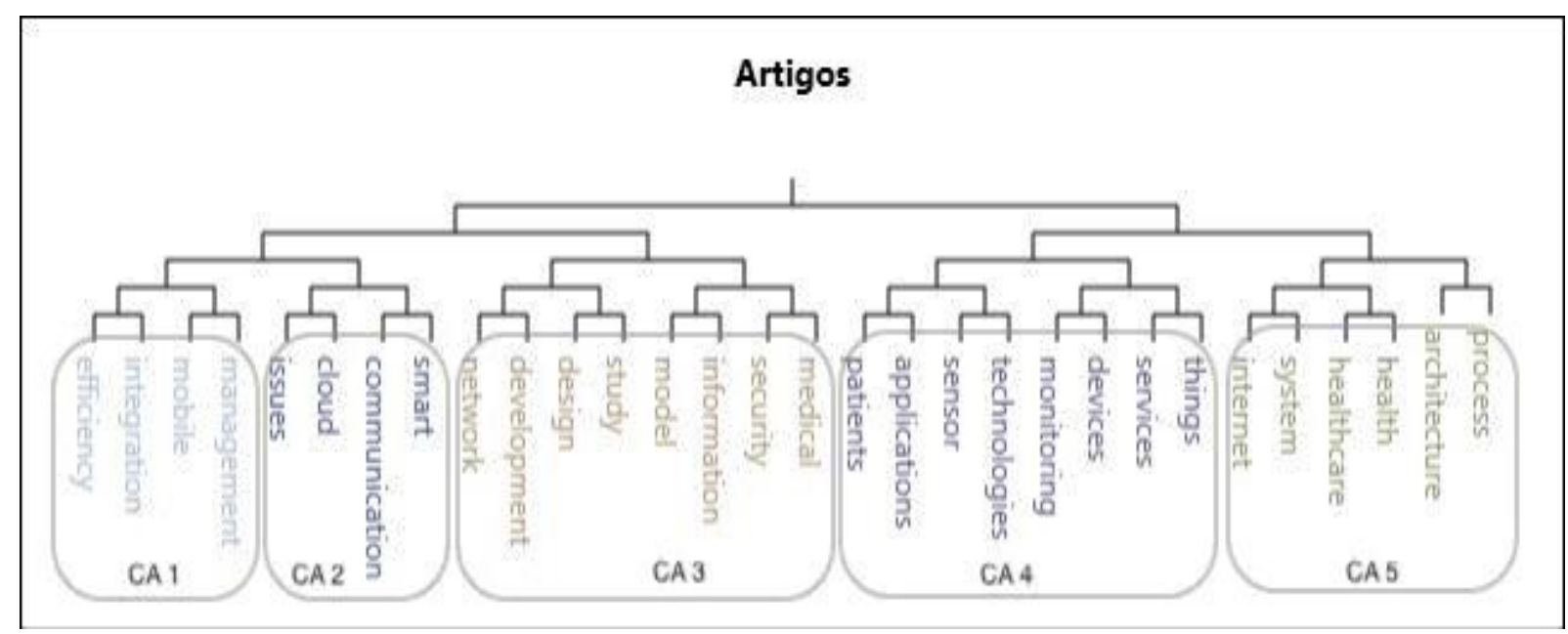

Fonte: Elaborado com base em Scopus.

Figura 2 - Agrupamento dos discursos comuns às patentes.

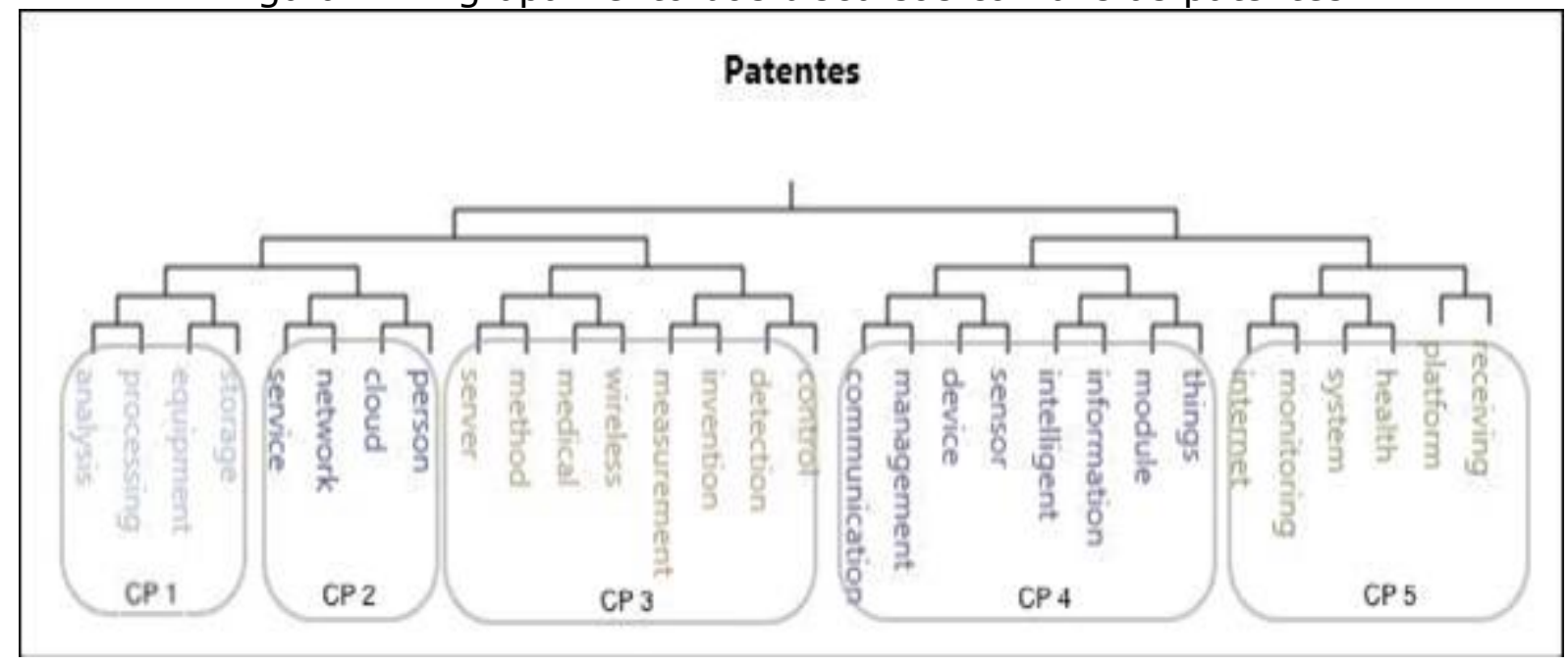

Fonte: Elaborado com base em Questel Orbit.

Ao analisar o agrupamento de discurso nota-se a convergência do discurso comum em artigos e patentes, demonstrando sinergia entre a produção científica e a produção tecnológica. Essa sinergia também é evidenciada na análise da frequência em que as palavras aparecem nos resultados analisados, destacando-se no conjunto de dados dos artigos: system (559); health (551), healthcare (504), internet (356), things (325), services (307), devices (265), monitoring (262), Technologies (257) e results (331), e no conjunto de dados das patentes: health (778), system (608), monitoring (464), internet(429), things (416), module (324), information (347), intelligent (313), management (295), sensor(254) e device (242). 
A partir da análise da correlação dos clusters de artigos (CA) e de patentes (CP) é possível delinear as principais tendências da IoT aplicada ao ambiente de saúde em nível global. Ao analisar os clusters CA1 e CP1 complementarmente a CA2 e CP2 identifica-se fortemente a abordagem das redes inteligentes de comunicação; armazenamento em nuvem; processamento, análise e gestão de dados, visando a integração e ganho de eficiência em serviços de saúde. Dentre as pesquisas que reforçam essa tendência destacam-se Hassanalieragh et al. (2015), Suciu et al. (2015), Feng (2016).

Em CA3 e CP3 identifica-se a abordagem da segurança e controle das informações geradas no ambiente da saúde, onde redes, métodos e modelos são estudados e desenvolvidos nesta perspectiva. Para Sun et al. (2018)os dispositivos médicos baseados em IOT geram em tempo real dados diversificados e altamente sensíveis que envolvem inclusive a privacidade e segurança do paciente,o que demanda a observação dos critérios de integridade, usabilidade, auditoria e privacidade no desenvolvimento de sistemas de segurança e privacidade desse tipo de dados.

A análise de CA4 com CP4 solidifica a principal tendência revelada neste estudo que são os dispositivos de monitoramento remoto através de sensores inteligentes integrados a rotina dos pacientes, considerado por Kang et al. (2018) como um benefício tangível da IoT no ambiente de saúde, uma vez que fornece soluções para problemas emergentes em serviços de saúde sobretudo no âmbito das doenças agudas e crônicas.

Ainda no contexto do monitoramento remoto de saúde, destaca-se em CA5 e CP5 a arquitetura de sistemas e plataformas integrados aos dispositivos inteligentes, que segundo Kang.et al., (2018)permitem a coleta de dados do paciente em tempo real e a transferência destes dados para avaliação por profissionais de saúde ou para o próprio paciente,na perspectiva de um monitoramento continuo de suas condições particulares de saúde.

\section{Conclusão}

O panorama delineado a partir dos indicadores bibliométricos indica que a produção científica e tecnológica envolvendo a temática da IoT no ambiente da saúde se desenvolveu a partir de 2010 liderada por países asiáticos,convergentes na publicação de artigos e patentes. A evolução histórica ocorreu de maneira equivalente até 2016 seguido por um crescimento acentuado na publicação de artigos.

A dispersão das publicações indica desconcentração da produção científica, com 4 artigos para o autor com maior produtividade e 12 publicações sobre a temática no principal periódico, destacando-se as áreas de Ciência da Computação e Engenharia, enquanto na produção 
tecnológica, mais concentrada, sobressai às áreas de Tecnologia Médica e Comunicação Digital.

A produção científica está predominantemente vinculada às Universidades, enquanto a produção tecnológica às empresas, entretanto há convergência no discurso comum presente nos artigos e patentes, apontando a tendência de inovações no âmbito da arquitetura de processos, sistemas e dispositivos relacionados a monitoramento remoto de pacientes através de sensores e plataformas inteligentes; redes de comunicação; armazenamento em nuvem; processamento, análise e gestão de dados de forma integrada e eficiente; segurança e controle de dados de saúde.

Cabe ressaltar que dentre os clusters identificados na análise e discussão dos dados, tanto em artigos quanto em patentes, o monitoramento remoto de pacientes foi evidenciado nos dois diferentes conjuntos de dados, o que demonstra uma sinergia entre a produção acadêmica e a geração de patentes em torno do tema.

Este estudo contribui na compreensão das características do conhecimento científico e tecnológico relacionado a temática da IoT aplicada a saúde, porém, constitui-se de uma "fotografia" do cenário atual. Ao considerar que o desenvolvimento científico e tecnológico não é estático, abre-se diferentes possibilidades de atualização deste quadro, inclusive na perspectiva do mapeamento de oportunidades derivadas do paradigma estudado, a partir das tendências já identificadas.

\section{Referências}

ALBERTIN, Alberto Luiz; ALBERTIN, Rosa Maria de Moura. A INTERNET DAS COISAS IRÁ MUITO ALÉM DAS COISAS. Gv-executivo, [s.l.], v. 16, n. 2, p.12-17, mar. 2017.

ARAUJO, Carlos Alberto. Bibliometria: evolução histórica e questões atuais. em Questão, Porto Alegre, v. 12, n. 1, p.11-32, 2006. Semestral.

ATUN, Rifat A. ; SHERIDAN, Desmond. INNOVATION IN HEALTH CARE: THE ENGINE OF TECHNOLOGICAL ADVANCES. International Journal Of Innovation Management. [s.I.], p. 5-10. jun. 2007.

BRIAN P. KALIS. Accenture (Ed.). Internet of Health Things Survey. 2017. Disponível em: https://www.accenture.com/t20170215t191150 w/usen/ acnmedia/pdf-42/accenture-health-2017-internet-of-health-thingssurvey.pdf. Acesso em: 12 out. 2018. 
ENGELMANN, Iomani. Saúde 4.0: a revolução no cuidado com o paciente. 2018. Disponível em: http://forumsaudedigital.com.br/saude-4-0revolucao-no-cuidado-com-o-paciente/. Acesso em: 10 out. 2018.

FENG, Nan. Research on the Modern Intelligent Healthcare Platform from the Perspectives of Grid based Cloud Com. International Journal Of Grid And Distributed Computing, [s.I.], v. 9, n. 11, p.35-48, 30 nov. 2016.

FIDLER, Bradley; CURRIE, Morgan. The Production and Interpretation of ARPANET Maps. Ieee Annals of The History of Computing, [s.I.], v. 37, n. 1, p.44-55, jan. 2015.

Financiadora de Estudos e Projetos - FINEP (Ed.). Manual de Oslo: Diretrizes para coleta e interpretação de dados sobre inovação. 2005. Disponível em: https://www.finep.gov.br/images/apoio-efinanciamento/manualoslo.pdf. Acesso em: 18 out. 2018.

FREITAS, Maria Helena de Almeida. Avaliação da produção científica: considerações sobre alguns critérios. Psicologia Escolar e Educacional, Campinas, v. 2, n. 3, p.211-228, 1998.

GIL, Antonio Carlos. Como elaborar projetos de pesquisa. 4. ed. São Paulo: Atlas, 2002. 175 p.

GIL, Antonio Carlos. Métodos e Técnicas de Pesquisa Social. 6. ed. São Paulo: Atlas, 2008. 200 p.

HASSANALIERAGH, Moeen et al. Health Monitoring and Management Using Internet-of-Things (IoT) Sensing with Cloud-Based Processing: Opportunities and Challenges. 2015 Ieee International Conference On Services Computing, [s.I.], p.285-292, jun. 2015.

KANG, Minhee et al. Recent Patient Health Monitoring Platforms Incorporating Internet of Things-Enabled Smart Devices. International Neurourology Journal, [s.l.], v. 22, n. 2, p.76-82, 31 jul. 2018

LAPLANTE, Phillip A.; LAPLANTE, Nancy. The Internet of Things in Healthcare: Potential Applications and Challenges. It Professional, [s.I.], v. 18, n. 3, p.2-4, maio 2016.

MAYERHOFF, Zea Duque Vieira Luna. UMA ANÁLISE SOBRE OS ESTUDOS DE PROSPECÇÃO TECNOLÓGICA. Cadernos de Prospecção, Salvador, v. 1, n. 1, p.7-9, 2008.

OLIVEIRA, Ely FrancinaTannuri de; GRACIO, Maria Cláudia Cabrini. Indicadores bibliométricos em ciência da informação: análise dos pesquisadores mais produtivos no tema estudos métricos na base 
Scopus. Perspectivas em Ciência da Informação, Marília, v. 16, n. 4, p.1628, dez. 2011.

RODRIGUES, Charles; VIERA, Angel Freddy Godoy. Estudos bibliométricos sobre a produção científica da temática Tecnologias de Informação e Comunicação. Incid: Revista de Ciência da Informação e Documentação, [s.l.], v. 7, n. 1, p.167-180, 5 abr. 2016.

SANTAELLA, Lucia et al. Desvelando a Internet das Coisas. Revista Geminis, [s.I.], v. 4, n. 2, p.19-32, 2013.

SANTOS, Thiago de Sousa; SOUZA, Paulo Augusto Ramalho de; PEREIRA, Raquel da Silva. PESQUISA EM TURISMO E DESENVOLVIMENTO REGIONAL: ANÁLISE DOS ESTUDOS REALIZADAS NO BRASIL ENTRE OS ANOS DE 1997 E 2013. XviiSemead: Seminários em Administração, São Paulo, p.1-14, out. 2014.

SILVA, Rogério Oliveira da; OLIVEIRA, José Lucas Sousa de. A INTERNET DAS COISAS (IOT) COM ENFOQUE NA SAÚDE. Tecnologia em Projeção, [s.l.], v. 8, n. 1, p.77-85, jan. 2017.

SPINAK, Ernesto. Indicadores cienciometricos. Ciência da Informação, Brasília, v. 27, n. 2, p.141-148, 1998.

SUCIU, George et al. Big Data, Internet of Things and Cloud Convergence - An Architecture for Secure E-Health Applications. Journal of Medical Systems, [s.l.], v. 39, n. 11, p.1-8, 7 set. 2015.

SUN, Wencheng et al. Security and Privacy in the Medical Internet of Things: A Review. Security and Communication Networks, [s.I.], v. 2018, p.1-9, 2018.

VERMESAN, Ovidiu; FRIESS, Peter (Org.). Internet of Things - Global Technological and Societal Trends. [s.I]: River Publishers, 2011. 316 p.

World Intellectual Property Organization (WIPO) (Org.). PCT - Sistema Internacional de Patentes. Disponível em: https://www.wipo.int/pct/pt/index.html. Acesso em: 28 nov. 2018. 